\title{
Perceptions Surrounding Mental Health in Young People and their Primary Caregivers and its Influence on Help-Seeking

\author{
Behaviour of Young People across Socio-Economic Groups in
}

\section{India}

\author{
Satvika $\operatorname{Char}^{1 *}$
}

\section{ABSTRACT}

The treatment gap that exists between the need and access of mental health care facilities is concerning and demands further enquiry. This exploratory research study aims to better understand barriers in accessing mental health care facilities for young people across socioeconomic groups in India through the following objectives: 1) To find out a young person's understanding and perception of mental health. 2) To understand if the participant has any stigma and perception surrounding mental health and mental disorders. 3) To understand if the participant's stigma, perception and understanding differs from that of their primary caregiver. 4) To understand the access and help-seeking behaviour of the participant to mental health care facilities and whether this is influenced by their own/primary caregiver's perceptions. A mixedmethods analysis was carried out. The total sample size of this study is 66 which includes 33 young people as primary participants and 33 primary caregivers as secondary participants. Quantitative data collection was carried out via a form through snowball and purposive sampling. Three scales were included- Kuppuswamy Socioeconomic Scale, Community Attitudes Toward the Mentally Ill Scale, and Attitudes Toward Seeking Professional Psychological Help Scale ( $\mathrm{N}=33$ ). The CAMI Scale was administered to the primary caregivers $(\mathrm{N}=33)$ of the young people participants as well. The participants were divided into 2 groups based on their score in the Kuppuswamy Socioeconomic Scale, Upper/Upper Middle $(\mathrm{N}=18)$ and Lower/ Upper Lower/ Lower Middle (N=15) This data was then analysed through SPSS using Kendall Tau's correlation coefficient and independent samples t-tests. Qualitative data was collected as a voluntary subset of the participants who filled out the quantitative data form, though a focus group discussion. 2 focus groups (Upper/Upper Middle and Lower/ Upper Lower/ Lower Middle) were interviewed through a semi-structured interview structure using an FGD Guide. This data was analysed using thematic analysis. The lowest and highest obtainable scores for the CAMI scale were 12 and 60 respectively. For the ATSPPH scale, these values were 0 and 30. The mean score for the young people CAMI was 45.09 ( $\mathrm{SD}=6.94)$. When the same scale was administered to the primary caregivers, the mean was 42.24 $(\mathrm{SD}=8.00)$. For the ATSPPH scale, the mean score was $19.06(\mathrm{SD}=6.65)$. The findings from

${ }^{1}$ Student, Krea University, India

*Corresponding Author

Received: October 31, 2021; Revision Received: November 28, 2021; Accepted: December 08, 2021

(C) 2021, Char S.; licensee IJIP. This is an Open Access Research distributed under the terms of the Creative Commons Attribution License (www.creativecommons.org/licenses/by/2.0), which permits unrestricted use, distribution, and reproduction in any Medium, provided the original work is properly cited. 


\section{Perceptions Surrounding Mental Health in Young People and their Primary Caregivers and its Influence on Help-Seeking Behaviour of Young People across Socio-Economic Groups in India}

this study suggest that stigma isn't the main barrier in help-seeking behaviour for the sample studied and that participants across socio-economic groups, without being influenced by the perceptions of their parents, have the desire to seek professional psychological help when experiencing mental distress. For the lower socio-economic category in particular, the problem lies in not knowing who to approach and in a lack of knowledge with regards to how to access available mental healthcare. The study also suggests that perceptions toward mental illness and the desire to seek professional psychological help do not vary significantly across socioeconomic groups.

Keywords: Perceptions and Stigma, Mental Health and Mental Illness, Young People, Primary Caregivers, Socio-Economic Groups, Help-Seeking Behaviour

$\mathrm{M}$ ental health is a matter of crucial importance and just like with any other physical illness, seeking help for mental illness is something that should be encouraged and supported. In India, the overall weighted prevalence for any mental morbidity is $13.7 \%$ in an individual's lifetime. Despite this, the treatment gap for mental disorders ranged between $70 \%$ and $92 \%$ for different disorders (Murthy, 2017).

A study examining a twelve-month prevalence and treatment gap for common mental disorders in India found the treatment gap to be 95\%, indicating that only 5 out of 100 individuals with common mental disorders received any treatment over the course of a year (Sagar et al., 2017). The socio-cultural context in India makes it so that the care of a mentally ill person here is primarily the responsibility of the family. The family may tolerate, protect, shield or reject the patient (Prasadarao, 2009). This behaviour could influence the seeking of professional help by the person experiencing mental distress, also referred to as help-seeking behaviour. This behaviour is defined as a problem focused, planned behaviour, involving interpersonal interaction with a selected health-care professional (Cornally \& McCarthy, 2011).

This can be a further heightened issue when the person in mental distress is a young person who might still be financially reliant on their family. Viewing the family as a place and family members as an ingroup induces Indians to resort to collectivist behaviour with collectivist intention (Sinha et al., 2001). The perceptions and understanding of mental health by primary caregivers, defined in this study as parents living with the young person or relatives or guardians living with the young person as extended family, could be a possible barrier towards help-seeking behaviour. The perceptions that the young person has of mental illness could also influence their own help-seeking behaviour as negative perceptions could lead them to believe that the society around them would feel similarly. This again could serve as a barrier to help-seeking behaviour.

The existence of stigma, in the minds of either the young person or their primary caregiver or both, could influence the help-seeking behaviour of the young person.

Keeping the economic context of India in mind is also important as the social and economic are both important factors and might interact with each other. In a developing country like India, there are a wide range of different socio-economic groups. Perceptions and understandings of mental illness might vary across these groups and so might the availability of mental healthcare resources, which in turn could affect help-seeking behaviour. This study therefore, aims to explore whether perceptions surrounding mental health in young people and 


\section{Perceptions Surrounding Mental Health in Young People and their Primary Caregivers and its}

Influence on Help-Seeking Behaviour of Young People across Socio-Economic Groups in India

their primary caregivers affect the help-seeking behaviour of young people aged 18-25, across socio-economic groups, in India.

\section{Concepts Studied}

The existence of stigma is defined according to Link and Phelan's definition that links component concepts under a broad umbrella concept they call stigma: "In our conceptualization, stigma exists when the following interrelated components converge. In the first component, people distinguish and label human differences. In the second, dominant cultural beliefs link labeled persons to undesirable characteristics - to negative stereotypes. In the third, labeled persons are placed in distinct categories so as to accomplish some degree of separation of "us" from "them." In the fourth, labeled persons experience status loss and discrimination that lead to unequal outcomes" (Link \& Phelan 2001, p. 367)

The different socio-economic groups will be defined in accordance with the 2021 updated version of the Kuppuswamy socio economic scale.

"Young People" are defined by World Health Organization (WHO) as an overlapping age group of 10 to 24 years which includes both 'adolescence'-age spanning 10 to 19 years and "youth"-those in 15-24 years of age. For the purpose of this study, the age group within the "young people" bracket is that of 18 to 24 .

In this study, primary caregivers are defined as parents living with the young person or relatives or guardians living with the young person as extended family.

\section{LITERATURE REVIEW}

While there have been studies examining individual aspects of the proposed research question, there is a dearth of studies that look at caregiver perceptions and young people perceptions toward mental health across socio-economic groups.

One study by Gronholm and colleagues in 2015 (N=407) conducted in Greater London looks at the role of caregiver characteristics in mental health service use by young people. The results showed that when caregivers' stigma-related characteristics were considered as a main effect, young people's likelihood of service use across health and education settings increased as caregivers reported less intended stigmatizing behaviours. However, when caregivers had used the service themselves, it was seen that caregivers' stigma-related characteristics were not associated with young people's services provided wholly or partially within health care services when caregivers had experience of service use themselves. The results from this study suggest that caregiver characteristics influence young people's use of services in a complex manner. Further research could help us understand better how stigma related factors and caregiver's experiences can influence help-seeking behaviour in young people.

In the Indian context, a study $(\mathrm{N}=521$, age 15-60) was conducted in 2014 by Gaiha and colleagues and aims to understand current levels of mental health literacy, including knowledge, information needs, perspectives, social attitudes, myths, probable actions and stigma related to mental illness of the community at large. Results showed that where to seek care was influenced deeply by culture and a lack of knowledge regarding the efficacy and availability of services by the DMHP. Although $91 \%$ of all respondents stated that mental illness could happen to anyone, $25 \%$ shared that they would keep the mental illness of a family member a secret from neighbours and friends. $33 \%$ of all respondents shared that a visit to a mental health care facility would entail the assumption that the person concerned has a mental 


\section{Perceptions Surrounding Mental Health in Young People and their Primary Caregivers and its Influence on Help-Seeking Behaviour of Young People across Socio-Economic Groups in India}

illness. Locking persons who appear to be mentally ill in a room till they act "normal" was shared by $16 \%$ of all respondents as a probable action. Even after recovery from mental illness, $18 \%$ of all respondents felt that they were not "fit" for employment.

Another study by Gaiha et al. (2020) conducted a systematic review on stigma associated with mental health problems among young people (aged 10-24) in India. The study showed that Indian youth lacked knowledge about the causes of mental illness, associated these with functional impediments and believed that limited/no treatment existed for such problems. They were also unable to identify common symptoms or use a common term or psychiatric label to describe the symptoms. People with mental health problems are perceived as dangerous and irresponsible, likely due to misinformation and misunderstanding of mental health problems as being solely composed of severe mental disorders. Young people in India also may not consider themselves vulnerable to acute problems or recognise everyday mental health problems when they experience them. Results showed that approximately $33 \%$ of the youth participants in 16 pooled studies had poor knowledge, $36 \%$ in 12 pooled studies had negative attitudes and $22 \%$ had stigmatizing behaviours in 4 studies. However, this metaanalysis showed a high degree of heterogeneity.

One study conducted by Saraf and colleagues in 2018 examined what adolescent girls living in an urban slum setting in India know about mental health through findings from a mental health literacy survey. The sample consisted of 337 women between the ages of 16 to 19 from low-income areas living in Bengaluru. Two vignettes on depression and self-harm were used. Results showed that in the first vignette, only $8 \%$ of women were able to label the condition as depression. Almost all the girls said that they would not consider seeking help from MHPs for various reasons. Stigma $(21.2 \%)$ was cited as a major reason by a majority of the respondents, followed by lack of information, fear, financial constraints, lack of belief in MHPs, misconceptions, and lack of proximity to a mental health clinic. Only a few considered mental health professionals as possible sources of help (19.3\% for depression and $2.4 \%$ for self-harm). Majority of participants felt that they would rather approach friends and parents as sources of help. The study showed that mental health literacy is low among young women from low-income areas.

Another study in Assam, India in 2020 by Basumatary and colleagues looked at magicoreligious beliefs, stigma and help-seeking behaviour among the caregivers of people with Schizophrenia. The study showed that the prevalence of stigma was $100 \%$. The study demonstrates strong belief in non-mental health professional support, particularly relying on magico-religious mediums for treating the persons with Schizophrenia. The results showed that supernatural beliefs and stigma were found to be common among the caregivers of persons with schizophrenia. The caregivers also demonstrated help-seeking tendencies from friends and family members. The caregivers have higher help-seeking behaviour from informal groups than mental health practitioners. The study focused only on quantitative measures and not qualitative data. A qualitative analysis would have given a more in-depth understanding of the reasoning behind these beliefs and lack of seeking support from mental health practitioners.

One qualitative study in India (Sadath et al., 2014) explored caregiving and help-seeking in first episode psychosis. The study aims to understand the caregivers' experiences and helpseeking behaviours for patients with FEP by trying to understand two areas, one being the delays in initiating appropriate psychiatric treatment for patients and the second being 


\section{Perceptions Surrounding Mental Health in Young People and their Primary Caregivers and its}

Influence on Help-Seeking Behaviour of Young People across Socio-Economic Groups in India

understanding the difficulties faced by the caregivers. Themes that emerged were, sequence of help-seeking wherein many caregivers took their ill relatives to faith healing practitioners, explanatory model of illness which suggested that the caregivers' perception of illness causation is associated with strong religious belief systems, Illness Management Strategies wherein the caregivers felt vulnerable and helpless, Financial Burden and Perceived Stress and Stigma. The study showed that the caregivers were hesitant to seek help due to their stigma attached to mental illness. The caregivers' poor understanding and high stigma caused substantial delays in seeking psychiatric treatment.

While there are studies that examine individual aspects of caregiver perceptions and young people perceptions toward mental health, there is a dearth of studies exploring the correlation between these two. Similarly, while there are studies that explore mental health perceptions in low-income groups, not many explore whether these perceptions differ from high income groups. Additionally, there is a lack of mixed method studies conducted on the same. Addressing this gap in research is important as it will give us an insight into if and how the perceptions of caregivers influence the perceptions and help-seeking behavior of the young people. A qualitative study with focus group discussions with both upper and lower socioeconomic groups will give a more nuanced perspective into whether perceptions and access to mental health care services differ across socio-economic groups in India.

\section{METHODOLOGY}

\section{Research Question}

Do perceptions surrounding mental health in young people and their primary caregivers influence help-seeking behaviour of young people aged 18-24 across socio-economic groups in India?

\section{This exploratory research study has the following objectives:}

1. To find out a young person's understanding and perception of mental health.

2. To understand if the participant has any stigma and perception surrounding mental health and mental disorders.

3. To understand if the participant's stigma, perception and understanding differs from that of their primary caregiver.

4. To understand the access and help-seeking behaviour of the participant to mental health care facilities and whether this is influenced by their own/primary caregiver's perceptions.

For objective 1, data collected through qualitative methods (FGD) will be studied. The use of qualitative methods will a deeper, more contextual understanding of what the participant understands about mental health.

For objectives 2 and 4, data collected both through qualitative (FGD) and quantitative methods (CAMI and ATSPPH Scales) will be studied. This approach will provide an insight into the participant's understanding of the variable as well as a deeper understanding into the context that surrounds this understanding.

For objective 3, data collected through quantitative methods (CAMI Scale) will be studied. Participant group consisting of the primary caregivers was asked to fill out the same questionnaire that was given to the participant group consisting of young people. 
Perceptions Surrounding Mental Health in Young People and their Primary Caregivers and its Influence on Help-Seeking Behaviour of Young People across Socio-Economic Groups in India

\section{Variables}

- Attitudes of young person toward the mentally ill

- Attitudes of primary caregiver toward the mentally ill

- Professional or Psychological Help-Seeking behaviour of the young person

\section{Sample and Sample Details}

For the purpose of this study, data was collected from 33 primary participants- the young people (aged 18 to 24), and 33 secondary participants- the respective primary caregivers. The total number of participants therefore, was 66.

The participant sample for this study is divided into 2 categories on the basis of the score on the Kuppuswamy Scale, subdivided into 4 sections.

Category 1 (Upper socio-economic group): Upper and Upper Middle socio-economic groups Section 1 are young people belonging to these groups. $(\mathrm{N}=18)$

Section 2 are the primary caregivers of the young people belonging to these groups. $(\mathrm{N}=18)$

Category 2 (Lower socio-economic group): Lower, Upper Lower and Lower Middle socioeconomic groups.

Section 3 are young people belonging to these groups. $(\mathrm{N}=15)$

Section 4 are the primary caregivers of the young people belonging to these groups. $(\mathrm{N}=15)$

\section{Method of Data Collection Quantitative Data}

The data was collected through snowball and purposive sampling. A google form which included the consent requirements, necessary scales and debriefing was circulated to the researcher's peers who then circulated it forward. People in parks and nearby localities in Mumbai were also approached and were asked to fill out printed copies of the form after informed consent was taken. As the participant sample included participants across socioeconomic groups, a language barrier had to be overcome. A translation agency was used to translate and back translate the scale in a regional language, Hindi.

\section{Qualitative Data}

In order to collect qualitative data, within the same form, the young people participants had the additional option to sign up for an online group discussion. 4 participants from each of the two socio-economic groupings (Category 1 and Category 2) signed up. However one participant from each group was unable to make it in the end owing to network or other disturbances. There were therefore 2 Focus Group Discussions using a semi-structured interview format each with 3 people. Before the FGD, an FGD Guide with 23 questions aimed at addressing objectives 1,2 and 4 of this study was developed. The FGD Guide too was forward and back translated into Hindi.

\section{Instruments Used}

Kuppuswamy Socio-Economic Scale: A modified version of this scale that had been adapted for the year 2021 by Shah and Sheikh. The Kuppuswamy scale, developed in 1976 is one of the most widely used indicators of socio-economic status. The Kuppuswamy SES has included 3 parameters and each parameter is further classified into subgroups and scores have been allotted to each subgroup which have been defined later in this paper. The total score of Kuppuswamy Scale ranges from 3-29 and it classifies families into 5 groups, "upper class, upper middle class, lower middle class, upper lower and lower socio-economic class." 


\section{Perceptions Surrounding Mental Health in Young People and their Primary Caregivers and its}

Influence on Help-Seeking Behaviour of Young People across Socio-Economic Groups in India

Community Attitudes Toward the Mentally Ill (CAMI) Scale: The first scale is a shortened 12 item subset adaptation developed in 2014 of the original 40 item Community Attitudes Toward the Mentally Ill (CAMI) Scale. This scale ascertains the levels of mental healthrelated stigma and tolerance answered via a 5-point Likert Scale ranging from 1 to 5 (1= strongly disagree, $5=$ strongly agree). The 12 CAMI statements were phrased in both positive and negative directions. Those relating to tolerance and support of community care expressed supportive attitudes towards mental illness where agreement was positive, while for those relating to prejudice and exclusion, disagreement reflected more positive views. A higher score represented a more positive attitude (less prejudiced/more tolerant). The lowest attainable score on this scale was 12 and the highest was 60.

Attitudes Toward Seeking Professional Psychological Help (ATSPPH): The second scale is a shortened 10 item adaptation of the Attitudes Toward Seeking Professional Psychological Help (ATSPPH). Items are rated on a 4-point Likert-type scale ( $3=$ Agree, $0=$ Disagree), where items 2, 4, 8, 9, and 10 are reverse scored. Scores are then summed together, with higher scores indicating more positive attitudes toward seeking professional help. The correlation between the 10-item short form and the original 29-item scale was 0.87 (Fischer and Farina, 1995). The lowest attainable score on this scale was 0 and the highest was 30 .

\section{Ethical Considerations}

Talking about topics such as mental health and stigma could lead to distress for the participants, therefore, as per section 8.05 of the APA code, participants were asked for informed consent via a form. The study was explained to the participants through a Participant Information Sheet. For the qualitative interviews, participants were asked to sign an informed consent form that stated that they would not disclose the identity of any of the other participants in their focus group. The consent form also provided additional details about where and how the data collected would be used. Participants were informed that their interviews would be transcribed by an external transcription agency who would ensure the confidentiality of their data.

Participants were also informed of their right to withdraw their consent at any point and the excision of this right would have been respected, had they chosen to withdraw consent. The participants were told that they should inform the researcher at any point if they felt uncomfortable. Participants were not deceived or misinformed in any way.

The participants were debriefed following the interview and were asked if they had any questions for the researcher. The confidentiality, anonymity and privacy of their data was ensured by changing names and concealing details that could help identify the participants and keeping the recordings and the transcripts in a password protected file.

\section{DATA ANALYSIS}

Triangulation was used as an analysis technique, utilizing more than one method of data collection and analysis in order to enhance validity, create a more in-depth picture of the research problem and to interrogate different ways of understanding the research problem.

The quantitative research method for this study involves descriptive and inferential statistics, with correlation and t-tests under the latter, used for the main analyses of the research questions. The Correlational test (Kendall tau's Correlation Coefficient) that was performed was a non-parametric test owing to the small sample size of the study. 
The Qualitative research involved a thematic analysis which involves a process of familiarization with the data following which patterns and themes are observed, reiteratively categorized and analysed.

\section{FINDINGS}

The main aim of the study was to determine whether there is any relationship between a young person's perception of mental illness, a primary caregiver's perception of mental illness and help-seeking behaviour of the young person. Additionally, the study also aimed to see if there is any difference in the above stated variables across socio-economic groups.

The results observed for the qualitative and quantitative analyses were as follows:

\section{Qualitative Analysis:}

The analysis of the qualitative data was done with the help of thematic analysis.

This analysis of the data yielded six themes as illustrated in Figure 1. These themes are being explored separately in order to analyse them in detail, however these themes are not entirely independent of one another. There are certain overlaps and interrelations between the themes presented as well as sub-themes that have been explored under the themes. Figure 1 outlines only the main themes.

\section{Figure 1}

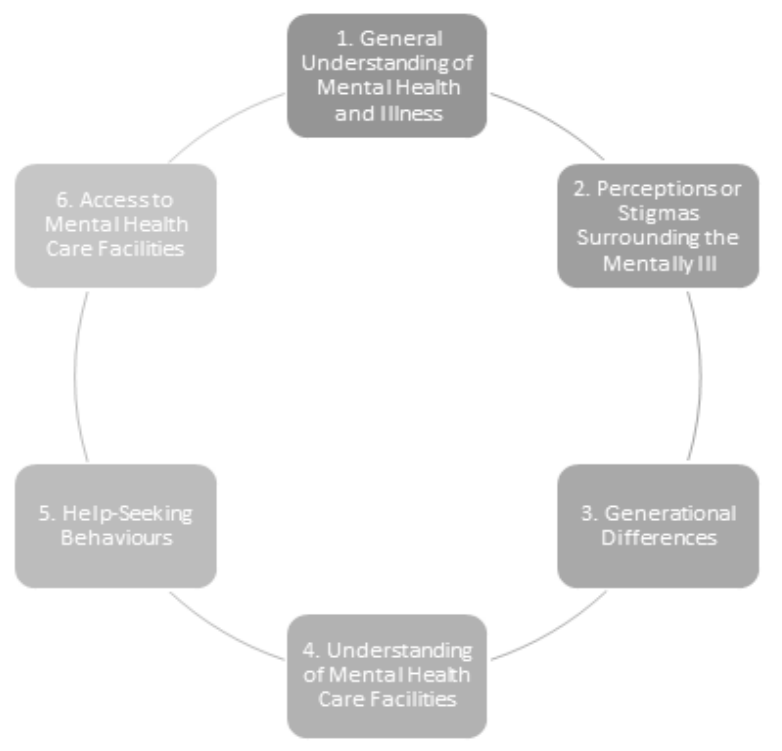

\section{Theme 1: General Understanding of Mental Health and Illness:}

This refers to the general understanding that the participants demonstrated on the areas of mental health and mental illness. Participants had slightly varying viewpoints of what mental health constitutes as well as their individual understandings of how they would try to provide health or support to someone who is mentally ill.

\section{- Sub-Theme 1: What mental health and illness constitutes:}

While participants from the upper socio-income group were more aware about how to define mental illness and mental health on a more general level, participants from the lower socioeconomic group had a vaguer understanding of the same and put forward certain pre-formed notions of what they believed mental illness would look like. The responses did not indicate stigma on the part of either group, however there were certain pre conceived notions of what mental illnesses look like. 


\section{- Sub-Theme 2: Idea of how to provide support:}

When the participants were asked what they would do if someone they knew told them that they were dealing with a mental illness, both sets of participants came forward with different perceptions of how they thought they should provide help.

Participant $\mathrm{H}$ from the upper socio-economic group shared that they would talk about what they think would help the person or what they felt the person approaching them wanted to hear.

While Participant K from the lower socio-economic group did suggest that they could suggest forms of mindfulness, the participant was also aware of their limited capacity to be able to contribute and provide help in a situation like this as opposed to doing what they felt would be right without a professional understanding of how to provide mental healthcare.

\section{Theme 2: Perceptions or Stigmas Surrounding the Mentally Ill:}

While there were positive perceptions surrounding those with mental illness or those who were seeking treatment for mental health conditions in both groups, there were also certain stigmas and perceptions that came through in the participants' responses. One common notion was that if someone is mentally ill, then there must be some cause behind it. Another perception that came up briefly among both groups was that someone could be using mental illness as a method of seeking attention. Both these sub-themes are explored below.

\section{- Sub-Theme 1: Reason Behind Mental Illness:}

Participants from both groups carried the notion that for a person to become mentally ill, there must be some reason that is causing them to feel this way.

Participant $\mathrm{H}$ from the upper socio-economic group carried this notion forward further and layered it by stating that a person's mental illness is caused due to their personalities and the kind of responses they might have to different situations. There was also this notion of a person having to see/struggle a lot in order for them feeling low to be justified.

Participant $\mathrm{K}$ from the lower socio-economic group stated that while it is very likely that mental illness is caused by a particular reason, also mentioned that it can very rarely happen that someone also experiences mental distress for no reason. Thus, this participant from the lower socio-economic group demonstrated an understanding that mental distress, while frequently has a cause behind it, does not necessarily adhere to having definitive reasons or causes of origin.

\section{- Sub-Theme 2: Attention Seeking Behaviour:}

Certain participants from both groups of the focus group discussion suggested that it is possible for a person to be faking their mental illness or exaggerating it as a means to gain attention or sympathy. This appeared to generally be determined on the basis of whether the person had a valid enough reason to be struggling or whether their struggle seemed genuine enough.

This indicates that the participant assumes that they would be able to determine the extent to which a person is mentally ill on the basis of his external behaviour.

This viewpoint is countered by Participant PS from the upper socio-economic group who states that it is possible that the notion of being misunderstood as looking for attention, or the pressure to fit into a particular manner of appearing and behaving to justify your mental distress might hold people back from being open about their struggles. 


\section{Theme 3: Generational Differences}

As this study seeks to also explore the differences between perceptions surrounding mental health across generations and look at whether the family's viewpoints influence those of the young people, the participants were asked about their family's understanding of mental health during the discussion.

\section{- Sub-Theme 1: Family's Understanding:}

When it comes to exploring the participants' family's understanding of mental health, the participants felt that their families were not very open to discussing mental health and they felt that they did have certain stigmas associated with it that they carried with them.

\section{- Sub-Theme 2: Youth and Progressive Thinking:}

The participants felt that there was a significant difference in the thought processes that were held by their family in comparison to their own beliefs when it came to mental health.

When asked if they felt like their family's understanding of mental health had influenced theirs in any way, Participant $\mathrm{N}$ from the upper socio-economic group stated that that had definitely not happened and that this participant was far more open about mental health than their family however this participant also stated that the family beliefs were gradually becoming more progressive.

While Participant $\mathrm{N}$ seemed to believe in the potential for dynamic and gradually changing thought processes even amongst family beliefs, Participant $\mathrm{R}$ from the lower socio-economic group believed that it was not possible for the thoughts of the older generations to change, however that it was possible for the newer generation to have their own independent way of thinking.

\section{Theme 4: Understanding of Mental Healthcare Facilities:}

The participants were asked about what they knew about mental health care facilities and seeking professional help at a time of mental distress. The participants displayed a certain lack of clarity regarding the same. The participants also had a notion of how they expected a mental health professional would be able to provide help or support to them.

- Sub-Theme 1: Knowledge:

The participants, when asked about the different mental health care facilities that were available to them, demonstrated a certain lack of awareness across both the upper and lower socio-economic groups. While the upper socio-economic group was slightly more aware of the broader kinds of mental health care professionals, they too were not aware about who to approach for what kind of issue or that different mental health care professionals could address different types of conditions.

The lower socio-economic groups had an even less clear understanding of mental health care facilities and stated that they had no idea about who to approach. Participant $\mathrm{K}$ from the lower socio-economic group mentioned that nobody has taught them anything about this and neither do they have the opportunity to see and learn from somewhere.

Participant $\mathrm{K}$ also further elaborated that this lack of awareness is due to the lack of knowledge that is being passed down about mental health care facilities, there is no place for them to learn about it from.

- Sub-Theme 2: Expectations:

Through the discussion the participants also shared the various expectations that they had from mental health professionals or in what manner they felt one would be benefitted or not by speaking to such a person. 


\section{Perceptions Surrounding Mental Health in Young People and their Primary Caregivers and its}

Influence on Help-Seeking Behaviour of Young People across Socio-Economic Groups in India

Participant $\mathrm{H}$ from the upper socio-economic group stated that they felt that speaking to a professional would enable them to be free of any form of baggage, they would be able to be themselves without having to hold up an image of themselves as a result of speaking to a professional who has never seen them before.

Participant R from the lower socio-economic group discussion brought up the same idea but supported a contrary viewpoint which stated that since this person is someone you have never met, it is difficult to be entirely free with them.

There is also the assumption that people would expect that their problems should be solved by the mental health professional and a lack of certainty surrounding this might hold them back from approaching them freely.

However Participant $\mathrm{R}$ does state that while it might initially be unnerving to speak to a professional about your mental health, speaking to them would be easier once you push past the initial hesitation.

\section{- Sub-Theme 3: Stigma in Sharing Experiences:}

Participants from both the focus group discussions shared their apprehensions about sharing their experience of going to a mental health care practitioner with the people around them out of fear of societal stigma and judgement.

Participant PS from the upper socio-economic group shared that even though they would want to tell people close to them, the thought that it might change their perception of them was scary. This indicates the existence of a conflict, between wanting to tell people and feeling afraid to do so, even with the people you are close to, out of fear of being looked at differently.

Participant $\mathrm{R}$ from the lower socio-economic group shared a similar sentiment and also pointed out that it is not possible for them to disclose seeking mental health care in the same ease or manner in which one can disclose having a physical health checkup done. There is also the perception in society that those who visit mental health care facilities are mad or unstable in a way.

\section{Theme 5: Help-Seeking Behaviour:}

The participants were also asked how they would handle their own mental distress, how they would set about getting help and who they would feel comfortable sharing their problems with. Participants discussed sharing their concerns with their family, friends and mental health care practitioners.

\section{- Sub-Theme 1: Approaching Friends and Family:}

While some participants shared that they would be comfortable speaking to their family, quite a few participants shared that they would find it difficult to do so.

Participant $\mathrm{N}$ from the upper socio-economic group stated that they do not share their mental health with their immediate family.

Participant $\mathrm{K}$ from the lower socio-economic group added on to this by saying that while there are certain issues you can share with your family, there are scenarios such as relationship troubles for instance where sharing with your family becomes difficult as they would simply not understand in a non-judgmental manner. 


\section{Perceptions Surrounding Mental Health in Young People and their Primary Caregivers and its}

Influence on Help-Seeking Behaviour of Young People across Socio-Economic Groups in India

When participants were asked if they felt more comfortable sharing with friends as compared to sharing their concerns with their families, there was a general consensus amongst both groups. Regardless of the levels of comfort, it appears that the first people that the participants approach in case of mental distress would be their friends or family depending on who they feel more comfortable sharing with.

\section{- Sub-Theme 2: Approaching a Mental Healthcare Practitioner:}

Participants were also asked to share their thoughts on seeking help from a mental health care practitioner and whether they would feel comfortable approaching one if they knew how to access them.

Participant $\mathrm{N}$ from the upper socio-economic group said they would first give it time and would approach a mental health care practitioner if they feel that their mental health is deteriorating further.

Participant R from the lower socio-economic group stated that they would first approach their close ones and if it felt like there was no other solution, they would definitely speak to a professional.

Participant $\mathrm{K}$ on the other hand held the view that, if possible, it is better to approach a mental health care professional directly than approaching your family first. They acknowledge that there might be a certain discomfort in approaching a professional however state that this discomfort exists even with approaching the family, therefore it is preferable to build up the level of comfort with a professional.

\section{Theme 6: Access}

The most prominent point of difference between the two focus groups, upper socio-economic and lower socio-economic, appeared at the discussion surrounding access and availability of mental health care facilities.

When the participants from the upper socio-economic group discussion were asked if they ever felt the need to approach a mental health care facility but found themselves unable to do so, all three participants said that they had never faced this problem.

On the other hand, when the participants of the lower socio-economic group were asked the same question, they provided multiple instances and reasons owing to which they were unable to approach necessary mental health care.

Participant $\mathrm{K}$ from the lower socio-economic group shared that there were occasions where they felt the need to approach a mental health care facility but did not know who to go to. Participant $\mathrm{R}$ from the lower socio-economic group stated that they do not have the right words to be able to describe the nature of the mental distress that they are dealing with and how there is a gap in the knowledge and understanding of this area.

While participant $\mathrm{R}$ stated that there are financial concerns involved with accessing mental health care facilities coupled with the uncertainty of whether the money is being put to good use, Participant K interjected saying that money isn't the biggest problem here. 
Participant K stated that even if there was the availability of free or affordable resources, the lack of awareness about these facilities would hold them back from going even when they really felt the need to do so.

\section{Quantitative Analysis}

A descriptive view of the overall scores from the 3 scales (CAMI, ATSPPH and Kuppuswamy Socioeconomic Scale) has been given in Table 1.

Table 1: Descriptive Statistics

\begin{tabular}{|l|l|l|l|l|l|l|}
\hline & N & $\begin{array}{l}\text { Min } \\
\text { Score } \\
\text { Obtained }\end{array}$ & $\begin{array}{l}\text { Max } \\
\text { Score } \\
\text { Obtained }\end{array}$ & Median & Mean & $\begin{array}{l}\text { Std. } \\
\text { Dev. }\end{array}$ \\
\hline CAMI (young people) & 33 & 26 & 56 & 46 & 45.09 & 6.94 \\
\hline CAMI (primary caregiver) & 33 & 22 & 54 & 45 & 42.24 & 8.00 \\
\hline ATSPPH & 33 & 3 & 27 & 22 & 19.06 & 6.65 \\
\hline $\begin{array}{l}\text { Kuppuswamy Socioeconomic } \\
\text { Scale }\end{array}$ & 33 & 3 & 29 & 20 & 17.73 & 8.97 \\
\hline
\end{tabular}

Table 1 shows that relatively higher means were observed for the CAMI Scale for both the young people and primary caregivers which indicates that a greater number of young people participants and primary caregiver participants had positive perceptions towards the mentally ill.

Initially, a correlation analysis using Kendall Tau's Correlation Coefficient was carried out between the three scales to determine whether there is a correlation between the young person's perception of mental illness, a primary caregiver's perception of mental illness and help-seeking behaviour of the young person (Table 2).

Table 2: Kendall Tau's Correlation Analysis

\begin{tabular}{|l|l|l|l|}
\hline Variables & $\mathbf{1}$ & $\mathbf{2}$ & $\mathbf{3}$ \\
\hline 1. CAMI (young person) & 1.00 & $0.255^{* *}$ & 0.069 \\
\hline 2. ATSPPH & $0.255^{* *}$ & 1.00 & 0.243 \\
\hline 3. CAMI (primary caregiver) & 0.069 & 0.243 & 1.00 \\
\hline
\end{tabular}

$* *$ significant $(\mathrm{p}<0.05)$

Results showed a positive association between the CAMI of the young person and ATSPPH. $(\mathrm{r}=0.255 ; \mathrm{p}<0.05)$.

This indicates that a more positive attitude towards the mentally ill and mental illness is seen along with a higher desire to seek professional psychological help in the case of mental distress. 
However, no association was found between the CAMI of the young person participants and the CAMI of the primary caregiver participants. This indicates that the perspectives of mental health for the caregiver have no association with the perspectives of the young person.

No correlation was found between the CAMI of the primary caregivers and the ATSPPH of the young people participants either. This indicates that there is a lack of association between the help-seeking behaviour of the young people participants and their primary caregivers' perception of mental health.

Independent sample t-tests were conducted for:

1. Socio-economic differences with respect to help-seeking behaviour (Table 3.1)

2. Socio-economic differences with respect attitudes of the young people participants toward the mentally ill (Table 3.2)

Table 3.1: Independent samples t-test for Socio-Economic Category with respect to ATSPPH Scale

\begin{tabular}{|c|c|c|c|c|}
\hline & \multicolumn{2}{|c|}{$\begin{array}{l}\text { Levene's Test for Equality of } \\
\text { Variances }\end{array}$} & \multicolumn{2}{|c|}{$\begin{array}{l}\text { t-test for Equality of } \\
\text { Means }\end{array}$} \\
\hline & $\mathbf{F}$ & Sig. & $\mathbf{t}$ & df \\
\hline $\begin{array}{l}\text { Equal } \\
\text { Assumed }\end{array}$ & 1.318 & 0.260 & 1.909 & 31 \\
\hline $\begin{array}{l}\text { Equal Variances Not } \\
\text { Assumed }\end{array}$ & & & 1.871 & 26.776 \\
\hline
\end{tabular}

The findings from the independent samples t-test showed that there was no significant difference found between help-seeking behaviour and socio-economic status.

This indicates that a particular socio-economic group of young people participants isn't necessarily more or less inclined towards having the desire to seek professional psychological help.

Table 3.2: Independent samples t-test for Socio-Economic Category with respect to CAMI Scale for young people participants

\begin{tabular}{|l|l|l|l|l|}
\hline & \multicolumn{2}{|l|}{$\begin{array}{l}\text { Levene's Test for Equality of } \\
\text { Variances }\end{array}$} & \multicolumn{2}{l|}{$\begin{array}{l}\text { t-test for Equality of } \\
\text { Means }\end{array}$} \\
\cline { 2 - 5 } & F & Sig. & t & df \\
\hline $\begin{array}{l}\text { Equal } \\
\text { Assumed Variances }\end{array}$ & 0.004 & 0.953 & 1.237 & 31 \\
\hline $\begin{array}{l}\text { Equal Variances Not } \\
\text { Assumed }\end{array}$ & & 1.254 & 30.959 \\
\hline
\end{tabular}

The findings from the independent samples t-test showed that there was no significant difference found between attitudes toward the mentally ill among the young people participants and socio-economic status. 


\section{Perceptions Surrounding Mental Health in Young People and their Primary Caregivers and its}

Influence on Help-Seeking Behaviour of Young People across Socio-Economic Groups in India

This indicates that a particular socio-economic group of young people participants isn't necessarily more prejudiced toward those with mental illness.

\section{DISCUSSION}

The study aimed to explore whether perceptions surrounding mental health and illness in young people aged 18-24 was influenced by the perceptions had by their primary caregivers with regards to the same, in India. Additionally, the study aimed to see whether these perceptions influenced the help-seeking behaviour of the young people and whether the desire to seek help varied across socio-economic groups.

A mixed-methods analysis was carried out. Quantitative data collection was carried out via a form through snowball and purposive sampling. Three scales were included- Kuppuswamy socio-economic Scale, Community Attitudes Toward the Mentally Ill Scale, and Attitudes Toward Seeking Professional Psychological Help Scale $(\mathrm{N}=33)$. The CAMI Scale was administered to the primary caregivers $(\mathrm{N}=33)$ of the young people participants as well. The participants were divided into 2 groups based on their score in the Kuppuswamy Socioeconomic Scale, Upper/Upper Middle (N=18) and Lower/Upper Lower/ Lower Middle $(\mathrm{N}=15)$ This data was then analysed through SPSS using Kendall Tau's correlation coefficient and independent samples t-tests. Qualitative data was collected as a voluntary subset of the participants who filled out the quantitative data form though a focus group discussion. 2 focus groups (Upper/Upper Middle and Lower/ Upper Lower/ Lower Middle) were interviewed through a semi-structured interview structure using an FGD Guide. This data was analysed using thematic analysis. The lowest and highest obtainable scores for the CAMI scale were 12 and 60 respectively. For the ATSPPH scale, these values were 0 and 30 . The mean score for the young people CAMI was 45.09 ( $\mathrm{SD}=6.94)$. When the same scale was administered to the primary caregivers, the mean was $42.24(\mathrm{SD}=8.00)$. For the ATSPPH scale, the mean score was $19.06(\mathrm{SD}=6.65)$.

The results from the qualitative analysis showed that the responses did not indicate stigma on the part of either group however there were certain preconceived notions of what mental illnesses look like. While participants from the upper/upper middle socio-economic group were able to define mental illness and health on a more general level, participants from the lower socio-economic groups had a vaguer understanding of the same. Both groups of participants had certain perceptions surrounding the mentally ill.

Participants from both groups carried the notion that for a person to be mentally ill, there must be some reason for them to feel this way. However, a participant from the lower/ upper lower/ lower middle socio-economic group left room for the possibility that mental distress can be experienced even for no particular reason. Certain participants from both groups suggested that it is possible for a person to be faking/exaggerating their mental illness as a means to gain attention or sympathy. However, one member from the upper/upper middle socio-economic group countered this by stating that such notions could be precisely why some people might hold back from sharing their struggles with mental health.

When participants were asked about their family's understanding of mental health, both groups of participants felt that their families were not very open to discussing mental health and had certain associated stigmas with regards to the same. Growing up surrounded by a family that does not understand or support mental illness could hold the young people back from sharing their own potential struggles with mental illness and could also lead to a certain internalised perception or stigma that they too learned to have toward mental illness which would then

(c) The International Journal of Indian Psychology, ISSN 2348-5396 (e)| ISSN: 2349-3429 (p) | 1373 


\section{Perceptions Surrounding Mental Health in Young People and their Primary Caregivers and its Influence on Help-Seeking Behaviour of Young People across Socio-Economic Groups in India}

have to be unlearned as they grew up. Both groups of participants felt that there was a significant difference in the perceptions that were held by their family in comparison to their own understanding when it came to mental health. The possibility for the gradual increase in progressive views within family members however, was supported by one member of the upper/upper middle socio-economic group. This indicates that even though the older generations might have more obsolete views surrounding mental health, the young people are not easily influenced by these beliefs and take their time to question right from wrong, make an effort to explore and educate themselves before formulating a concrete opinion.

Both participants displayed a certain lack of clarity with regards to mental health care facilities but this lack of awareness was much greater in the participants of the lower/lower middle socio-economic groups. Participants expressed certain plus points and apprehensions about seeking help from a professional. One participant from the upper/upper middle socioeconomic group suggested that since the mental health professional is a neutral third party, the participant would be able to be themself. A participant in the lower/ upper lower/ lower middle socio-economic group expressed that it might be initially difficult to open up to a stranger. Participants from both the focus groups shared their apprehensions about discussing their experience of going to a mental health care practitioner with the people around them and expressed a fear of societal stigma and judgement. Finding it difficult to share with the people around them or the community one is a part of that they are accessing mental health care can make it difficult to access mental health care in the first place.

When participants were asked how they would handle their own mental distress, how they would set about getting help and who they would feel comfortable sharing their problems with, while some participants were comfortable with speaking to their families, quite a few participants from both groups shared that they would find this difficult. There was a general consensus among both groups that the first people that the participants would approach in case of mental duress would be either their friends or family. When participants were asked whether they would like to seek help from a mental health practitioner, the general perception indicated that it is easier and more comfortable to approach your family and closer circle first as opposed to directly seeking help from a professional but that if the participants felt the need to do so and had the resources available, they would go ahead and approach a professional as well.

The biggest point of difference between the two focus groups was with regards to the access and availability of mental health care facilities. Participants from the upper/upper middle socio-economic groups could not think of an instance where they had felt the need to approach a mental health care facility but found themselves unable to; however, participants from the lower/ upper lower/ lower middle socio-economic group provided multiple instances of wanting to but not being able to access mental health care. A participant from the latter group expressed those financial concerns were an inhibiting factor in accessing these facilities. Another participant from the same group expressed that even if there was the availability of free or affordable resources, the lack of awareness about these facilities would hold them back from going even when they really felt the need to do so. The perspectives put forth by the participants from the lower socio-economic category outline the various problems that they have been facing in terms of accessing mental health care facilities, be it knowledge, terminology, money or availability, which is not the case with the members of the upper socioeconomic groups. 


\section{Perceptions Surrounding Mental Health in Young People and their Primary Caregivers and its Influence on Help-Seeking Behaviour of Young People across Socio-Economic Groups in India}

The quantitative analysis shows us that higher means were obtained in the CAMI scale of the young person indicating less stigma/more positive perceptions. This is seen in the qualitative discussions as well where the young people participants express positive attitudes toward mental health and those with mental illness. There is a significant positive correlation $(\mathrm{p}<0.05)$ between the attitudes/perceptions that a young person has toward the mentally ill (CAMI Scale) and their attitudes toward seeking professional psychological help (ATSPPH Scale). This is corroborated further by the qualitative discussions where participants across both socio-economic groups demonstrated an understanding of the importance of seeking mental healthcare. There was no significant correlation between the CAMI scores of the young people and the CAMI scores of the primary caregiver. This is supported through the qualitative data wherein participants clearly stated that they don't find themselves being influenced by the attitudes of their parents/primary caregivers and find that they have more progressive attitudes toward mental health.

There was no correlation between the CAMI Scores of the primary caregivers and the ATSPPH scores of the young person. This too is supported by the qualitative analysis that suggests that young people recognise the importance of seeking professional help for mental distress and express a distinct difference from the views of their parents/primary caregiver with regards to mental health. This more positive attitude is seen through the descriptive data as well where the young people show a slightly higher mean, 45.09 and lesser SD 6.94 than that of their primary caregivers $(\mathrm{M}=42.24, \mathrm{SD}=8.00)$. The fact that the difference in the two mean scores isn't very much can be further understood through the qualitative data that suggests that the views of the primary caregivers too seem to be slowly and gradually becoming more progressive.

The findings from the independent samples t-test for socio-economic category with respect to CAMI Scale for young people showed that there was no significant difference between attitudes toward the mentally ill and socio-economic status. This is supported by the qualitative analysis where it is seen that there is no drastic difference in the attitudes the participants from each group had toward mental illness and those that are mentally ill.

The findings from the independent samples t-test for socio-economic category with respect to ATSPPH Scale showed that there was no significant difference between help-seeking behaviour and socio-economic status. This finding is heavily resonated in the qualitative analysis where both groups, regardless of socio-economic status express that they would want to seek professional psychological help in case their mental health became unmanageable for them. The findings there showed that the main barrier to accessing mental health support was not a lack of desire or stigma but rather a lack of information with respect to how to access mental healthcare.

These findings are in support of certain pre-existing literature that states that one of the most significant barriers to accessing mental healthcare facilities is lack of information (Gaiha et al., 2014), this study indicates that participants have positive perceptions toward mental illness and do not carry with them a great degree of stigma surrounding the same. Certain other studies (Gaiha et al., 2020) indicate a high degree of stigma. However, the meta-analysis did show a high degree of heterogeneity. This difference could be because of the small sample size of this study as well as the fact that the population was predominantly living in urban locations. It might be interesting to see the difference in perfections across geographical locations in India in future research. 


\section{Perceptions Surrounding Mental Health in Young People and their Primary Caregivers and its Influence on Help-Seeking Behaviour of Young People across Socio-Economic Groups in India}

The findings from this study suggest that stigma isn't the main barrier in help-seeking behaviour and that participants across socio-economic groups, without being influenced by the perceptions of their parents, have the desire to seek help at times of mental distress. The problem lies in not knowing who to approach or how to set about accessing available mental healthcare, which is predominantly observed in the lower socio-economic category. The study also suggests that perceptions toward mental illness and the desire to seek professional psychological help do not vary significantly across socio-economic groups. The relatively high means for the attitudes toward the mentally ill held by the primary caregivers indicate that it is possible that even an older generation might not be carrying a high degree of stigma surrounding mental health with them which might be interesting to explore through further research.

The findings from the study give an insight into further research that can be done using more comparative groups to see the difference in perceptions toward mental health and help-seeking behaviour. Additionally, since this was a cross-sectional study, more research should be done that is longitudinal in nature and explores whether these perceptions and attitudes are subject to changing over time.

\section{CONCLUSION}

When a thematic analysis was carried out on the qualitative data, the following broad themes and sub-themes emerged: 1) General Understanding of Mental Health and Illness; i) What mental health and illness constitutes ii) Idea of how to provide support 2) Perceptions or Stigmas Surrounding the Mentally Ill; i) Reason Behind Mental Illness ii) Attention Seeking Behaviour 3) Generational Differences; i) Family's Understanding ii) Youth and Progressive Thinking 4) Understanding of Mental Healthcare Facilities; i) Knowledge, ii) Expectations iii) Stigma in Sharing Experiences 5) Help-Seeking Behaviour; i)Approaching Friends and Family ii) Approaching a Mental Healthcare Practitioner 6) Access

Results from the Kendall Tau's correlation analysis showed a positive association between the CAMI of the young person and ATSPPH $(r=0.255 ; \mathrm{p}<0.05)$. However, no association was found between the CAMI of the young person and CAMI of the primary caregiver. Additionally, no association was found between the CAMI of the primary caregiver and the ATSPPH of the young person. The findings from the first independent samples t-test (Table 3.1) showed that there was no significant difference found between help-seeking behaviour and socio-economic status. The findings from the second independent samples t-test (Table 3.2) showed that there was no significant difference found between attitudes toward the mentally ill among the young people participants and socio-economic status.

Findings from both the qualitative and quantitative analysis indicate that perceptions and stigma toward mental illness do not vary significantly across socio-economic groups and that there is no significant association between the perceptions toward mental health that are held by the primary caregiver and the young people participants. Additionally, the study indicates that the prominent barrier to seeking professional psychological help in the case of mental distress for the lower socio-economic category is not stigma or a lack of desire to seek help but rather a lack of knowledge and means of accessing mental health care facilities.

\section{Limitations}

While the findings from this study are definitely insightful and pave the way for further research, it is important to acknowledge the drawbacks and shortcomings that this study had. 


\section{Perceptions Surrounding Mental Health in Young People and their Primary Caregivers and its Influence on Help-Seeking Behaviour of Young People across Socio-Economic Groups in India}

The findings from this study should be interpreted with caution as they might not be representative of the larger group that they are a part of. The sample size was comparatively small which limits generalizability. Since this was an exploratory study, it can only be suggested that there was a relationship between the variables. However, this does not establish causation. As the quantitative data was collected through the method of snowball sampling, it is possible that the participants who were closer to the researcher answered differently as compared to those who weren't. Since some of the forms were filled out over google and some face to face, this could have led to a certain difference in the manner or honesty with which the participants filled out the forms. The focus group discussions were held through online measures and without video to make the discussions between the upper/upper middle and lower/ upper lower/ lower middle groups more uniform, however this misses out on rich nonverbal behavioural context that could be gathered in an in person set up.

\section{REFERENCES}

Adolescent health and development. WHO Regional office for South-East Asia. [accessed on June 16, 2021]. Available from: http://www.searo.who.int/entity/child_adolescent/topics/adolescent_health/en/index. html

Basumatary, M., Ali, A., \& Daimari, B. N. (2020). Magicoreligious Beliefs Stigma and Helpseeking Behaviour among the Caregivers of Persons with Schizophrenia. National Journal of Professional Social Work, 21(1), 19-26

Chadda, R. K., Agarwal, V., Singh, M. C., \& Raheja, D. (2001). Help seeking behaviour of psychiatric patients before seeking care at a mental hospital. Int J Soc Psychiatry, 47, $71-78$.

Cornally, N., \& McCarthy, G. (2011). Help-seeking behaviour: A concept analysis. International journal of nursing practice, 17(3), 280-288.

Mathur Gaiha, S., Ann Sunil, G., Kumar, R., \& Menon, S. (2014). Enhancing mental health literacy in India to reduce stigma: the fountainhead to improve help-seeking behaviour. Journal of Public Mental Health, 13(3), 146-158. doi:10.1108/jpmh-06-2013-0043

Gaiha, S.M., Taylor Salisbury, T., Koschorke, M. et al. (2020). Stigma associated with mental health problems among young people in India: a systematic review of magnitude, manifestations and recommendations. BMC Psychiatry 20, 538, https://doi.org/10.1186/s12888-020-02937-x

Gronholm PC, Ford T, Roberts RE, Thornicroft G, Laurens KR, Evans-Lacko S (2015) Mental Health Service Use by Young People: The Role of Caregiver Characteristics. PLoS ONE 10(3): e0120004. https://doi.org/10.1371/journal.pone.0120004

Hammer, J. H., Parent, M. C., \& Spiker, D. A. (2018). Mental Help Seeking Attitudes Scale (MHSAS): Development, reliability, validity, and comparison with the ATSPPH-SF and IASMHS-PO. Journal of Counseling Psychology, 65(1), 74.

Henderson, Helen., Henderson, Claire., Ilic, Nevena., Evans-Lacko, Sarah., \& Thornicroft, Graham. (2014) Attitudes towards Mental Illness - NHS Digital., healthsurvey.hscic.gov.uk/media/37730/HSE2014-Ch3-Attitudes-towards-mentalillness.pdf

James, S., Chisholm, D., Murthy, R. S., Kumar, K. K., Sekar, K., Saeed, K., \& Mubbashar, M. (2002). Demand for, access to and use of community mental health care: Lessons from a demonstration project in India and Pakistan. Int J Soc Psychiatry, 48, 163-176.

Khandelwal, S. K., Jhingan, H. P., Ramesh, S., Gupta, R. K., \& Srivastava, V. K. (2004). India mental health country profile. International Review of Psychiatry, 16(1-2), 126-141. doi:10.1080/09540260310001635177 


\section{Perceptions Surrounding Mental Health in Young People and their Primary Caregivers and its}

Influence on Help-Seeking Behaviour of Young People across Socio-Economic Groups in India

Link, B.G., and Phelan, J.C. (2001). Conceptualizing stigma. Annual Review of Sociology, 27:363-385,

Murthy R. S. (2017). National Mental Health Survey of India 2015-2016. Indian journal of psychiatry, 59(1),

21-26.

https://doi.org/10.4103/psychiatry.IndianJPsychiatry_102_17

Prasadarao, P. S. D. V. (2009). International perspectives on culture and mental health. Culture and mental health: Sociocultural influences, theory, and practice, 149177.

Sadath, A., Muralidhar, D., Varambally, S., Jose, J. P., \& Gangadhar, B. N. (2014). Caregiving and Help Seeking in First Episode Psychosis: A Qualitative Study. Journal of Psychosocial Rehabilitation and Mental Health, 1(2), 47-53. doi:10.1007/s40737-0140013-2

Sagar, R., Pattanayak, R. D., Chandrasekaran, R., Chaudhury, P. K., Deswal, B. S., Lenin Singh, R. K., Malhotra, S., Nizamie, S. H., Panchal, B. N., Sudhakar, T. P., Trivedi, J. K., Varghese, M., Prasad, J., \& Chatterji, S. (2017). Twelve-month prevalence and treatment gap for common mental disorders: Findings from a large-scale epidemiological survey in India. Indian journal of psychiatry, 59(1), 46-55. https://doi.org/10.4103/psychiatry.IndianJPsychiatry_333_16

Saleem, Sheikh \& Jan, Shahsumaya. (2021). Modified Kuppuswamy socioeconomic scale updated for the year 2021. Indian Journal of Forensic and Community Medicine. 8. 13. 10.18231/j.ijfcm.2021.001.

Saraf, G., Chandra, P. S., Desai, G., \& Rao, G. N. (2018). What Adolescent Girls Know about Mental Health: Findings from a Mental Health Literacy Survey from an Urban Slum Setting in India. Indian journal of psychological medicine, 40(5), 433-439. https://doi.org/10.4103/IJPSYM.IJPSYM_108_18

Sinha, J. B. P., Sinha, T. N., Verma, J., \& Sinha, R. B. N. (2001). Collectivism coexisting with individualism: an Indian scenario. Asian Journal of Social Psychology, 4(2), 133-145. doi:10.1111/j.1467-839x.2001.00081.x

Srivastava, V. K. (2004). India mental health country profile. Int Rev Psychiatry, 16, 126141.

\section{Acknowledgements}

The author would like to thank Dr. Mercian Daniel of The George Institute for Global Health, under whose mentorship and guidance she authored this research paper. The author appreciates all those who participated in the study and helped facilitate the research process.

\section{Conflict of Interest}

The author(s) declared no conflict of interest.

How to cite this article: Char S. (2021). Perceptions Surrounding Mental Health in Young People and their Primary Caregivers and its Influence on Help-Seeking Behaviour of Young People across Socio-Economic Groups in India. International Journal of Indian Psychology, 9(4), 1359-1378. DIP:18.01.130.20210904, DOI:10.25215/0904.130 